\section{B THYMOSIN $\beta 4$ - A NOVEL REGULATOR OF LOW DENSITY LIPOPROTEIN RECEPTOR RELATED PROTEIN 1 (LRP1) IN VASCULAR DISEASE}

'Sonali Munshaw*, 'Susann Bruche, ${ }^{2}$ Jyoti Patel, ${ }^{1}$ Andia Redpath, ${ }^{3}$ Karina N Dubé, ${ }^{4}$ Regent Lee, ${ }^{4}$ Ashok Handa, ${ }^{2}$ Keith M Channon, ${ }^{1}$ Nicola Smart. ${ }^{1}$ Burdon Sanderson Cardiac Science Centre, Department of Physiology, Anatomy \& Genetics, University of Oxford, Sherrington Building, South Parks Road, Oxford OX1 3PT, UK; ${ }^{2}$ BHF Centre of Research Excellence, Division of Cardiovascular Medicine, John Radcliffe Hospital, University of Oxford, UK; ${ }^{3}$ UCL-Institute of Child Health, 30 Guilford Street, London WC1N 1EH, UK; ${ }^{4}$ Nuffield Department of Surgical Sciences, University of Oxford, Oxford, UK

\subsection{6/heartjnl-2019-BCS.225}

Vascular stability and tone are maintained by contractile smooth muscle cells (VSMCs). Injury-induced growth factors stimulate a phenotypic switching of VSMCs, from their quiescent contractile state to a more active synthetic phenotype that proliferate and migrate. Chronic VSMC dedifferentiation leads to vascular thickening and stiffness, exacerbates inflammation and promotes atherosclerotic lesion development and susceptibility to abdominal aortic aneurysm (AAA). Inhibiting VSMC phenotypic transformation has thus been shown to attenuate progression of vascular disease. We previously identified Thymosin $\beta 4$ (T $\beta 4)$ as a key regulator of embryonic VSMC differentiation. TMSB4X, encoding $\mathrm{T} \beta 4$, is the most abundant transcript in healthy and AAA aorta, therefore we hypothesised that T $\beta 4$ may additionally function to maintain vasculature and protect against disease throughout postnatal life. We identified an interaction between $\mathrm{T} \beta 4$ and Low density lipoprotein receptor related protein 1 (LRP1), an endocytic regulator of PDGFR? signalling which controls VSMC proliferation. LRP1 variants have been identified by GWAS as major risk loci for AAA and coronary artery disease. Adult T $\beta 4$-null mice displayed aortic VSMC and elastin defects, phenocopying LRP1 mutants and suggesting compromised vascular integrity. During development, $\mathrm{T} \beta 4$ functions in a paracrine manner, secreted from endothelial cells (ECs) to induce mesoderm to VSMC differentiation. To distinguish between cell-autonomous and paracrine roles for $\mathrm{T} \beta 4$, and simultaneously discern adult maintenance versus developmental requirements, we selectively induced deletion of $\mathrm{T} \beta 4$ from VSMCs or ECs at 3 weeks of age. Histological assessment of aortas at 12 weeks demonstrated that VSMC-specific $\mathrm{T} \beta 4$ knockdown recapitulated the global KO phenotype, revealing a postnatal requirement for $\mathrm{T} \beta 4$ to maintain healthy vasculature. In keeping with this, we confirmed predisposition of these mice to disease in models of atherosclerosis and AAA. Aneurysmal aorta and plaques of T $\beta 4 \mathrm{KO}$ were characterised by accelerated contractile-synthetic VSMC switching, elastin degradation and augmented PDGFR? signalling. In vitro, enhanced sensitivity to PDGF$\mathrm{BB}$, upon loss of $\mathrm{T} \beta 4$, coincided with increased cell surface recycling of LRP1-PDGFR? complexes and reduced lysosomal targeting, suggesting that dysregulated endocytosis underlies enhanced phenotypic switching and proliferation. Given the VSMC differentiation, anti-inflammatory and anti-apoptotic roles of $\mathrm{T} \beta 4$, we sought to determine the vasculoprotective potential of exogenous T $\beta 4$. In the AAA model, T $\beta 4$ treatment significantly reduced aortic dilatation and rupture, and preserved VSMC phenotype and elastin integrity, associated with normalisation of PDGFR? signalling. Our study identifies T $\beta 4$ as a key regulator of LRP1 for maintaining vascular health, providing insight that may reveal useful therapeutic targets for modulation of VSMC phenotypic switching and disease progression.

\section{IDENTIFICATION OF THE MAJOR GENETIC CONTRIBUTORS TO TETRALOGY OF FALLOT}

1,2Donna J Page* ${ }^{3,4}$ Matthieu J Miossec, ${ }^{1}$ Simon G Williams, ${ }^{1}$ Richard M Monaghan, ${ }^{1}$ Elisavet Fotiou, ${ }^{4}$ Heather J Cordell, ${ }^{4}$ Louise Sutcliffe, ${ }^{4}$ Ana Topf, ${ }^{5,6}$ Mathieu Bourgey, ${ }^{5}$ Guillaume Bourque, ${ }^{5}$ Robert Eveleigh, 7,8Sally L Dunwoodie, 9,10,11 David S Winlaw, ${ }^{12}$ Shoumo Bhattacharya, ${ }^{13}$ Jeroen Breckpot, ${ }^{14}$ Koenraad Devriendt, ${ }^{14}$ Marc Gewillig, ${ }^{15}$ David Brook, ${ }^{15}$ Kerry Setchfield, ${ }^{16}$ Frances A Bu'Lock, ${ }^{17}$ John O'Sullivan, ${ }^{18}$ Graham Stuart, ${ }^{19}$ Connie Bezzina, ${ }^{19}$ Barbara JM Mulder, ${ }^{20,21}$ Alex V Postma, ${ }^{22}$ James $R$ Bentham, ${ }^{23}$ Martin Baron, ${ }^{24}$ Sanjeev S Bhaskar, ${ }^{24}$ Graeme C Black, ${ }^{24}$ William G Newman, ${ }^{25}$ Kathryn E Hentges, ${ }^{5}$ Mark Lathrop, ${ }^{4}$ Mauro Santibanez-Koref, ${ }^{1}$ Bernard D Keavney. ${ }^{1}$ Division of Cardiovascular Sciences, School of Medical Sciences, Faculty of Biology, Medicine, and Health, Manchester Academic Health Science Centre, University of Manchester, Manchester, M13 9PT, UK; ${ }^{2}$ School of Healthcare Science, Manchester Metropolitan University, Manchester, M1 5GD, UKi ${ }^{3}$ Center for Bioinformatics and Integrative Biology, Faculty of Biological Sciences, Universidad Andrés Bello, Santiago, Chile; ${ }^{4}$ Institute of Genetic Medicine, Newcastle University, Central Parkway, Newcastle-upon-Tyne, NE1 3BZ, UK; ${ }^{5}$ McGill Genome Center, Montréal, QC, Canada; ${ }^{6}$ Canadian Centre for Computational Genomics, Montréal, QC, Canada; ${ }^{7}$ Faculties of Medicine and Science, University of New South Wales, Sydney, NSW, Australia; ${ }^{8}$ Chain Reaction Program in Congenital Heart Disease Research, Victor Chang Cardiac, Research Institute, Sydney, NSW, Australia; ${ }^{9}$ School of Child and Adolescent Health, Sydney Medical School, University of Sydney, Australia; ${ }^{10}$ Victor Chang Cardiac Research Institute, Australia; ${ }^{11}$ Heart Centre for Children, The Children's Hospital at Westmead, Sydney, NSW, Australia; ${ }^{12}$ RDM Cardiovascular Medicine, Wellcome Centre for Human Genetics, University of Oxford, Oxford, OX3 7BN; ${ }^{13}$ Center for Human Genetics, Catholic University Leuven, Leuven, Belgium; ${ }^{14}$ Pediatric and Congenital Cardiology, UZ Leuven, Leuven, Belgium; ${ }^{15}$ School of Life Sciences, University of Nottingham, Queen's Medical Centre, Nottingham, NG7 2UH; ${ }^{16}$ Honorary Associate Professor in Congenital and Paediatric Cardiology, East Midlands, Congenital Heart Centre and University of Leicester, Glenfield Hospital, Leicester, LE3 9QP; ${ }^{17} \mathrm{MD}, F R C P I$, Adult Congenital and Paediatric Cardiac Unit, Freeman Hospital, Newcastle upon Tyne; ${ }^{18}$ University Hospitals Bristol NHS Foundation Trust, Bristol UK; ${ }^{19}$ Heart Center, Department of Clinical and Experimental Cardiology, Academic Medical Center, Amsterdam, the Netherlands; ${ }^{20}$ Department of Medical Biology, Academic Medical Center, Amsterdam, the Netherlands; ${ }^{21}$ Department of Clinical Genetics, Academic Medical Center, Amsterdam, the Netherlands; ${ }^{22}$ Department of Paediatric Cardiology, Yorkshire Heart Centre, Leeds, UKi ${ }^{23}$ Division of Molecular \& Cellular Function, School of Biological Sciences, Faculty of Biology Medicine, and Health, Manchester Academic Health Science Centre, University of Manchester, Manchester, M13 9PL, UK; ${ }^{24}$ Manchester Centre for Genomic Medicine, Saint Mary's Hospital, Oxford Rd, Manchester M13 9WL; ${ }^{25}$ Division of Evolution and Genomic Sciences, Faculty of Biology, Medicine and Health, Manchester Academic Health Science Centre, University of Manchester, Oxford Road, Manchester, M13 9PT, UK

\subsection{6/heartjnl-2019-BCS.226}

There is strong evidence from familial recurrence studies for a genetic predisposition to sporadic, non-syndromic Tetralogy of Fallot (TOF). TOF is the most common, cyanotic congenital heart disease (CHD) phenotype yet the cause for the majority of cases remains elusive. Rare genetic variants have been identified as important contributors to the risk of $\mathrm{CHD}$, but relatively small numbers of TOF cases have been studied to date. 829 TOF patients underwent whole exome sequencing (WES), the largest cohort of non-syndromic TOF patients reported to date. The prevalence of unique, deleterious variants was determined; defined by their absence in the Genome Aggregation Database (gnomAD) and a scaled combined annotationdependent depletion (CADD) score of $\geq 20$. Clustering analysis of variants revealed that two genes, NOTCH1 and FLT4, surpassed thresholds for genome-wide significance (assigned as $\mathrm{P}<5 \times 10^{-8}$ ), after correction for multiple comparisons. NOTCH1 was most frequently found to harbour unique, deleterious variants. 31 variants were observed in 37 probands (4.5\%; 95\% confidence interval [CI]: $3.2-6.1 \%)$ and included 
seven loss-of-function variants, 22 missense variants and two in-frame indels. Sanger sequencing of the unaffected parents of seven cases identified five de novo variants. Three NOTCH1 variants (p.G200R, p.C607Y and p.N1875S) were subjected to functional evaluation and two showed a reduction in Jagged1-induced NOTCH signalling. FLT4 variants were found in 2.4\% (95\% CI:1.6-3.8\%) of TOF patients, with 21 patients harbouring 22 unique, deleterious variants. The variants identified were distinct to those that cause the congenital lymphoedema syndrome Milroy disease. In addition to NOTCH1, FLT4 and the well-established TOF gene, TBX1, we identified potential association with variants in several other biologically plausible candidate genes. In summary, the NOTCH1 locus is the most frequent site of genetic variants predisposing to non-syndromic TOF, followed by FLT4. Together, variants in these genes are found in almost $7 \%$ of TOF patients.

\section{STRESS MYOCARDIAL OXYGENATION AND NOT PERFUSION RESERVE DETERMINES ARRHYTHMIC RISK IN HYPERTROPHIC CARDIOMYOPATHY: INSIGHTS FROM A NOVEL OXYGEN-SENSITIVE CMR APPROACH}

${ }^{1}$ Betty Raman** ${ }^{1}$ Kenneth Chan, ${ }^{1}$ Masliza Mahmod, ${ }^{1}$ Moritz Hundertmark, ${ }^{1}$ Rina Ariga, ${ }^{1}$ Sanjay Sivalokanathan, ${ }^{2}$ Theodoros Karamitsos, ${ }^{3}$ Joseph Selvanayagam, ${ }^{1}$ Aaron Hess, ${ }^{1}$ Elizabeth M Tunnicliffe, ${ }^{1}$ Hugh Watkins, ${ }^{1}$ Stefan Neubauer*. ${ }^{1}$ Division of Cardiovascular Medicine, Radcliffe Department of Medicine, University of Oxford, Oxford, UK; ${ }^{2}$ First Department of Cardiology, Aristotle University of Thessaloniki, AHEPA Hospital, Thessaloniki, Greece; ${ }^{3}$ Department of Cardiovascular Medicine, Flinders Medical Centre, Bedford Park, Adelaide. Australia

10.1136/heartjnl-2019-BCS.227

Myocardial ischemia has long been implicated in promoting arrhythmic events and triggering sudden cardiac death in hypertrophic cardiomyopathy (HCM). However, the evidence for this is scarce due to challenges presented in direct ischemia assessment which generally requires an invasive approach. Blood oxygen level dependent cardiac magnetic resonance (BOLD CMR) permits the non-invasive assessment of tissue oxygenation, without gadolinium contrast, overcoming many limitations suffered by traditional methods. From a clinical perspective, T2-prepared steady-state free precession (T2-SSFP) BOLD is promising, but suffers from reduced diagnostic accuracy, owing to imprecisions in BOLD measurements secondary to heart-rate (HR) dependence. To resolve this, we developed a novel oxygen-sensitive CMR approach, Fast Low Angle Shot (FLASH) interleaved T2-SSFP BOLD, which was designed to eliminate both HR dependence and spatial variations seen with standard T2-SSFP BOLD. A comparison of both standard and novel approaches in 20 healthy subjects confirmed that FLASH-normalised T2-SSFP BOLD is highly reproducible, HR independent and more precise than standard T2-SSFP BOLD. In addition, the mean BOLD effect did not differ between the two methods. Importantly, using this novel approach, one could visualise changes in oxygen-sensitive signal from rest to stress qualitatively, making it feasible for direct incorporation into a clinical work flow. We then set out to test the hypothesis that stress oxygenation (as assessed on FLASH normalised T2-SSFP BOLD) is more powerful that myocardial perfusion reserve (MPRI) at determining arrhythmic risk in HCM patients. Adenosine stress BOLD, first pass perfusion imaging and late gadolinium enhancement CMR were undertaken in 103 genotyped-HCM patients. All patients underwent 24-hour Holter to monitor for evidence of ventricular tachycardia $(\geq 3$ beats, $\geq 120$ beats per minute). Thirty-two age- and sexmatched healthy subjects served as controls. Although both stress oxygenation and MPRI were impaired in HCM, only stress oxygenation, but not MPRI, associated with ventricular tachycardia on univariate analysis. There was a step-wise increase in ventricular tachycardia prevalence with decreasing quartiles of stress oxygenation. HCM patients with the lowest quartile of oxygenation were at a three-fold increased risk of ventricular tachycardia (OR 3.04, $\mathrm{p}=0.04$ ) after adjusting for LGE mass, age and baseline risk of sudden cardiac death. Sarcomeric mutation status was an independent determinant of stress oxygenation, irrespective of the extent of hypertrophy, MPRI or LGE burden (univariate predictors). In conclusion, we have successfully developed and implemented a novel oxygen-sensitive CMR method which has provided important insights into the role of stress oxygenation as a promising biomarker of arrythmic risk and potential therapeutic target for drug discovery in HCM. 